\title{
The Continued Quest for Pediatric Readmission Risk Prediction
}

\author{
Lauren G. Solan, MD, MEd', Katherine A. Auger, MD, MSc ${ }^{2,3,4 *}$
}

\begin{abstract}
'Division of Pediatric Hospital Medicine, Golisano Children's Hospital at the University of Rochester Medical Center, Rochester, New York;

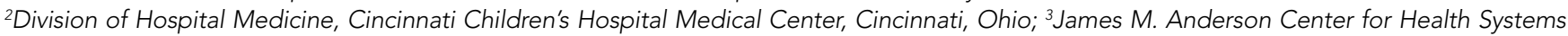
Excellence, Cincinnati Children's Hospital Medical Center, Cincinnati, Ohio; ${ }^{4}$ Department of Pediatrics, University of Cincinnati College of Medicine, Cincinnati, Ohio.
\end{abstract}

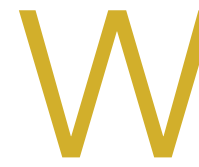

7 hile the use of pediatric readmission rates as a quality metric remains controversial, pediatric hospital-to-home transitions need improvement. ${ }^{1}$ As many as one-third of pediatric readmissions are preventable, ${ }^{2}$ but the multifactorial and complex nature of factors that contribute to pediatric readmissions presents a challenge in tackling readmission. Several factors are associated with increased risk of readmission; these factors include both clinical and sociodemographic characteristics, ${ }^{3}$ however, we still have much to learn. Further, the only large trial of an intervention to prevent pediatric readmissions across all comers (nontargeted) was unsuccessful in decreasing reutilization. ${ }^{4}$ By contrast, various studies have succeeded in reducing readmission and/or emergency department revisit rates associated with inpatient interventions in select populations. ${ }^{5}$ Currently, however, no standardized or validated pediatric risk prediction tool can reliably identify the high-risk patients who may benefit from interventions. In the Journal of Hospital Medicine, Brittan and colleagues add to the literature base exploring the factors associated with an increased 30-day readmission risk by trialing an electronic health record (EHR)-based tool composed of three components: presence of home health, polypharmacy in the form of $\geq 6$ medications, and presence of a caregiver who prefers a language other than English. ${ }^{6}$

This brief report contributes significantly to the literature. First, the presence of a tool embedded within the pediatric EHR and readily accessible at the point of clinical care is novel. Study authors purposefully chose components easily extractable from the EHR which update automatically. This infrastructure generates an automated score that is easily accessible to clinicians in real-time. Second, the transparency of the tool is notable given its display via the EHR's "Discharge Readiness Report" where a clinician can see not only the total compos ite score (one point for each component) but also the specific components for which a point was allocated. Although a composite score in and of itself is potentially helpful, understanding specific factors that contribute to a patient's increased risk

*Address for correspondence: Katherine A. Auger, MD, MSc, 3333 Burnet Ave, MLC 9016, Cincinnati OH 45220, 513.803.3234; Telephone: 513-803-3234; Fax: 513-803-9244; E-mail: Katherine.auger@cchmc.org

Published online first August 29, 2018.

Received: June 7, 2018; Accepted: June 20, 2018

(c) 2018 Society of Hospital Medicine DOI 10.12788/jhm.3071 of readmission allows for better targeting of interventions. For example, in Brittan's simple, three-component model, the presence of polypharmacy might trigger a pharmacist to meet with the family prior to discharge to discuss indications for and how to properly administer medications. Finally, a multidisciplinary team composed of clinicians, nurse-family educators, case managers, social workers, and informatics experts developed and implemented this tool. Although the rollout and longitudinal use of this tool is not described, the engagement of these multiple provider types is likely to increase successful rollout and utilization of the tool.

Unfortunately, the utility of this tool in predicting readmission is limited as evidenced by its low c-statistic. This limitation may be due to several reasons. The tool was not originally built as a tool to predict readmissions but rather an instrument to identify complex discharge care as part of a quality improvement initiative to improve discharge processes. Given the questions about readmission risk prediction, the authors explored the potential for the tool to predict readmission risk. The authors acknowledge that the tool excluded many known readmission risk factors based upon inconsistent documentation within the EHR and the desire to emphasize only modifiable factors. Thus, variables, including prior hospitalization which is a well-documented risk factor for readmissions (but not modifiable) and social determinants of health (which are not consistently documented), were excluded. Additionally, the included variable of "language preference" may have been a considerably broad characteristic. Limited English proficiency has been increasingly recognized as a construct placing patients at higher risk for adverse outcomes. However, caregivers with high-English proficiency also exhibit varying degrees of health literacy. The inclusion of health literacy may be additive to a readmission risk prediction tool. Finally, the outcome is not described well with regard to identification of "unplanned" events. Thus, their outcome measure may have included planned admissions for which the readmission risk prediction tool would be irrelevant.

In summary, Brittan and colleagues engaged a multidisciplinary group of providers to address discharge planning processes and leveraged the EHR to support their efforts in the form of a brief screening tool. Although this tool was not predictive of hospital readmissions, it highlights the opportunity to better utilize the EHR to gather meaningful, real-time data and subsequently use this information to positively impact our clinical care and allocation of resources. The tool should serve as a stepping stone to building a more extensive tool 
with inclusion of other known and potential readmission risk factors, thus resulting in a clinically relevant readmission risk prediction tool.

Disclosures: The authors have nothing to disclose.

\section{References}

1. Solan LG, Beck AF, Brunswick SA, et al. The family perspective on hospital to home transitions: a qualitative study. Pediatrics 2015;136(6):e1539-1549. doi: 10.1542/peds.2015-2098.

2. Toomey SL, Peltz A, Loren S, et al. Potentially preventable 30-Day hospital readmissions at a children's hospital. Pediatrics. 2016;138(2). doi: 10.1542/ peds.2015-4182.

3. Berry JG, Hall DE, Kuo DZ, et al. Hospital utilization and characteristics of patients experiencing recurrent readmissions within children's hospitals. JAMA. 2011;305(7):682-690. doi: 10.1001/jama.2011.122.

4. Auger KA, Simmons JM, Tubbs-Cooley $\mathrm{H}$, et al. Hospital to home outcomes $(\mathrm{H} 2 \mathrm{O})$ randomized trial of a post-discharge nurse home visit. Pediatrics. In press.

5. Auger KA, Kenyon CC, Feudtner C, Davis MM. Pediatric hospital discharge interventions to reduce subsequent utilization: a systematic review. J Hosp Med. 2014;9(4):251-260. doi: 10.1002/jhm.2134.

6. Brittan MS, Martin SL, Anderson, Moss A,Torok MR. An electronic health record tool designed to improve pediatric hospital discharge has low predictive utility for readmissions. J Hosp Med. 2018:13(11):779-782. doi: 10.12788/ jhm.3043. 